\title{
A study of emotionalism in patients undergoing rehabilitation following severe acquired brain injury
}

\author{
Joanna McGrath \\ Rivermead Rehabilitation Centre, Abingdon Road, \\ Oxford OX4 1XD, UK \\ Tel.: +44 1865 255426; Fax: +44 1865200185
}

\begin{abstract}
The present study describes the phenomenon of emotionalism in a sample of brain injured patients of mixed aetiology, with a view to identifying issues relevant to clinical management, and possible causal factors. 82 subjects with severe acquired brain injury undergoing rehabilitation participated in a structured interview in which they were asked to report the presence/absence of emotionalism and degree of distress associated with it. Their overt crying behaviour was also observed and recorded. Independent variables that predicted crying during the interview were identified using a multiple logistic regression procedure. Prevalence rates of emotionalism-tearfulness were high in this sample (52\% on basis of self-report, $36-41 \%$ on basis of observed behaviour). Emotionalism-laughter was much less common (13\%). Emotionalism-tearfulness was usually accompanied by negative affect, occurred in response to identifiable precipitants, and was often controllable. It was associated with major personal distress in about half the subjects who reported it. Independent variables which predicted crying behaviour were female gender and focal damage to the right cerebral hemisphere. It is concluded that an increased readiness to cry is common in people with severe acquired brain injury of mixed aetiology. The behaviour is meaningful, though not always distressing. The intensity of the behaviour is variable, and it may be most appropriate to regard emotionalism as a dimension rather than a syndrome. Implications for clinical management are discussed.
\end{abstract}

Keywords: Emotionalism, stroke, head injury, lability, crying, distress

\section{Introduction}

The phenomenon of emotionalism following brain injury or disease is a good example of a behavioural pattern that is perhaps unique to neurological patients, and is not addressed by current psychiatric classification systems. It is also sometimes referred to as 'emotional lability', 'inappropriate emotion', 'emotional incontinence', 'pathological crying or affect', and 'pseudobulbar affect'. It has been described in a wide range of neurological disorders including multiple sclerosis [22], motor neurone disease [7], cerebral tumour [24], epilepsy [20], Alzheimer's disease [11], and stroke [9]. Its main clinical feature is an increased readiness to cry or, more rarely, laugh [2]. There is no agreed definition of the behaviour involved, and this is reflected in the many names that exist for it, and in the variability of clinical descriptions in the literature. Some authors refer to uncontrollable crying which occurs with little or no warning [10]; others refer to crying that does not appear to be precipitated by an external event, or is precipitated by a trivial external event [19]; others refer to crying that is not accompanied by negative affect [17].

A number of different brain areas have been implicated. The earliest cases reported involved damage to subcortical structures including the brainstem, diencephalon, and medial temporal lobes [24]. The term 'pseudobulbar affect' has been used to describe emotionalism associated with bilateral damage to bulbar motor nuclei [12]. House and colleagues found an association between large lesions to the left frontal and temporal lobes and emotionalism in a community stroke sample [9]. Other authors have reported single cases demonstrating emotionalism in the presence of right frontal lesions [4,8], and argued for a causal link between right frontal lobe damage and emotionalism [19, 18]. In addition, fluoxetine [21], imipramine [4], and L-dopa [3] have all been found to be effective in treating cases of emotionalism, suggesting that different neural pathways and transmitters may be involved in different cases. Emotionalism may therefore be a non-specific sign of brain damage, or it may be that this class of 
behaviour can be broken down into specific syndromes each associated with different lesion sites. At present there is no clear evidence to support the latter hypothesis. However, clinical impression suggests qualitative differences in emotionalism between patients whose strokes involve the cerebral cortex and those whose strokes are largely confined to the brainstem.

Systematic careful descriptions of the phenomenology of emotionalism are rare, and confined to studies of stroke patients. House and colleagues note that, while emotionalism is clearly distinct from depression, there was a good degree of co-morbidity in their sample of stroke patients [9]. Thus many of their subjects who cried were also experiencing negative affect. Crying tended to occur in 'emotional' contexts and was therefore not inappropriate but, in their terminology, disinhibited. Allman reported on a sample of 30 stroke patients who showed emotionalism. $83 \%$ of subjects had some warning that they were about to cry, and felt that they could control the crying to some extent. The crying occurred in circumscribed contexts, and was most usually elicited by thoughts of family or the illness, kind gestures, the arrival or departure of visitors, television or music, disagreements, and the inability to perform a task $[1,3]$. Thus it appears that, for this population at least, emotionalism is largely provoked rather than spontaneous, accompanied by negative affect, occurs with some warning, and is excessive rather than inappropriate.

The prevalence of emotionalism in the Oxford community stroke sample is reported as $15 \%$ at one month, $21 \%$ at six months, and $12 \%$ at one year following stroke [10]. However, this study employed quite a strict definition of emotionalism (increased episodes of uncontrollable crying with little or no warning) so this is likely to be a conservative estimate. Rates are also likely to be higher than this in hospital samples [3], who are usually at an earlier stage of recovery or with more significant brain injury than community samples.

The present study arose in the course of a larger project investigating the emotional response to acquired brain injury in a sample of in-patients undergoing rehabilitation. The focus of the main project was fear and anxiety, and one of the key questions was whether subjects with acquired brain injury can reliably identify distinct emotional states, rather than experiencing generic distress. Subjects therefore participated in a structured interview [13] in which they were asked about a range of 10 emotional experiences or behaviours (Apathy; Irritability; Frustration; Fear; Sadness; Inappropriate behaviour; Worry; Emotionalism-tearfulness;
Emotionalism-laughter; Confusion). These interview items were derived from pilot studies in which subjects were asked open ended questions about their emotional status, but are also broadly consistent with the literature (e.g. $[14,15])$.

As the project progressed the tendency for subjects to cry during the assessment interview was so striking that further investigation of the phenomenon became an important endeavour in its own right. The behaviour was clearly identifiable to the observer, seemed to occur more frequently than might be expected on the basis of the literature, was socially awkward for both interviewer and subject, and seemed to signify profound distress.

The objectives of the present study were therefore to establish the prevalence of emotionalism in this sample; to identify environmental precipitants of emotionalism; to investigate the degree to which it was associated with significant distress; to explore its relationship with other affective states; and to identify patient characteristics which were associated with emotionalism (for instance neurological diagnosis, degree of disability or lesion location). A better understanding of this phenomenon can form the basis for more effective clinical management.

\section{Methods}

\subsection{Setting}

The study took place in a 25 bedded Regional unit specialising in the early rehabilitation of adults suffering from non progressive damage to the central nervous system, most of whom are admitted directly from district general hospitals or specialist neuroscience centres.

\subsection{Sample}

105 patients consecutively admitted to unit, and undergoing their first period of rehabilitation, were approached and asked to participate in a structured interview. Eighty two (78\%) subjects completed the interview. Of the 23 subjects who did not participate in the interview 15 subjects had cognitive problems involving language or attention which limited their comprehension; 6 subjects were capable but did not consent; 1 subject was acutely ill; 1 subject completed only a portion of the interview. Sample characteristics are 
Table 1

Characteristics of Sample

\begin{tabular}{ll}
\hline$n=82$ & \\
\hline Age & meange 17-70 46.76 \\
& median 47 \\
Gender & males 52 \\
& females 30 \\
Diagnosis & stroke 40 \\
& (left lesion 17, right lesion 19) \\
& head injury 23 \\
& subarachnoid haemorrhage 8 \\
& tumour 2 \\
& other (e.g., anoxia) 9 \\
\hline
\end{tabular}

Table 2

Characteristics of non-participants

\begin{tabular}{ll}
\hline$n=23$ & range 19-66 \\
Age & mean 47.47 \\
& median 48 \\
& males 9 \\
Gender & females 14 \\
& stroke 9 \\
Diagnosis & (left lesion 4, right lesion 2) \\
& head injury 6 \\
& subarachnoid haemorrhage 5 \\
& other 3 \\
\hline
\end{tabular}

given in Table 1 with characteristics of non-participants presented for comparison purposes in Table 2.

Of the 40 subjects with a diagnosis of stroke, C.T. scan reports indicated the presence of left cerebral hemisphere lesions in 17 (42\%) and right cerebral hemisphere lesions in $19(48 \%)$. All but one of the head injured subjects had suffered severe head injuries (posttraumatic amnesia ranged from 3 to 52 weeks, with a median of 10 weeks).

\subsection{The interview}

Subjects were interviewed in a consulting room during the second week of their admission to the unit. The interviews were tape recorded and transcribed at a later time. If a subject cried during the interview the investigator offered to terminate the conversation, but all subjects requested to continue the interview. Some complained that most social interactions were terminated when they cried, and they were therefore glad to have an opportunity to carry on with the conversation despite their tears.

The interview covered the 10 items in turn, first asking subjects if they had experienced the emotion or behaviour in the previous week and then, for those subjects who were capable of more than reliable yes/no responses (as assessed by the unit speech and language therapist), a series of supplementary questions. Thus, the portion of the interview which concerned emotionalism contained the following questions, which referred to the previous week:

a) 'I would like you to tell me if you have been too emotional, that is crying or laughing too easily or for no good reason'

b) 'Have you noticed that any particular thing starts you feeling this way?'

c) 'Do you do things to help you cope with this feeling, or to make it go away, or to prevent it from starting?'

d) 'How upsetting is this feeling?':

$\begin{array}{ll}\text { Not upsetting } & 0 \\ \text { A bit upsetting } & 1 \\ \text { Very upsetting } & 2 \\ \text { Extremely upsetting } & 3\end{array}$

Prevalence rates of emotionalism in this sample were defined in three ways: the number of subjects who responded 'yes' to question a); the number of subjects who rated their level of distress at 2 or 3 in response to question d); and the number of subjects who wept during the interview (defined by the necessity of interrupting the interview to wipe their eyes).

\subsection{Factors associated with emotionalism}

Case notes and standardised assessment schedules routinely administered to all patients in the unit were used to identify factors which might predict emotionalism at interview (see Appendix). These variables related to diagnosis, time since onset, cognitive or motor impairment, neuropsychological status, level of functional disability, life events, prior psychiatric history, demography, and site of brain damage. Information on all these areas was available because the subjects underwent intensive multidisciplinary assessment during their admissions, which usually lasted several months. Information concerning lesion location and size was, however, limited and relatively imprecise. C.T. scan reports were available for $90 \%$ of subjects and M.R.I. scans were available for a small minority of these. However, due to time and resource limitations, the original films were not examined. Information on the side of focal damage was based on the written reports alone. 


\subsection{Analysis}

Responses to question b) and c) were subjected to content analysis, using two independent raters, one of whom as blind to the purpose of the study, to ensure reliability of both the emergent categories and the assignment of responses to these categories.

The relation between emotionalism and other emotional experiences examined by the interview was investigated by carrying out simple non parametric correlations (Kendall's tau, two tailed version) using the scores obtained for question d) with respect to each of the 10 items.

Finally, the characteristics of those subjects who wept during the interview were investigated using a multiple regression procedure to identify independent variables that predicted this behaviour. As the dependent variable was categorical (wept during interview versus did not weep during interview) a logistic regression procedure was used. This procedure is appropriate for use with independent variables that are categorical or measured using interval or ratio scales. Twenty independent variables were entered into the model (see Appendix).

Forward stepwise logistic regression, in which variables are added to the model in order of statistical significance, was used. The whole regression model was evaluated using the likelihood ratio (L.R.) test, and non predictive variables were removed on the basis of this statistic until a final best fit model was obtained. The statistical package employed was S.P.S.S. $6.0^{\mathrm{m}}$.

\section{Results}

\subsection{Prevalence rates}

Forty three of the 82 subjects interviewed (52\%) answered 'yes' to the question concerning an increased readiness to cry in the previous week. $13(16 \%)$ answered 'yes' to the corresponding question concerning laughter.

The number of subjects who rated their distress levels as 'very' or 'extremely' distressed were 21 (26\%) for tearfulness and $1(2 \%)$ for laughter.

Thirty of the 82 subjects interviewed cried during the procedure. In addition, 6 subjects who did not consent to be interviewed said that they were too upset to participate and wept during this conversation. Thus, 36 out of 88 subjects approached $(41 \%)$ cried during the interview or pre-interview procedure.

All those subjects who cried during the interview had also responded 'yes' to the question concerning increased readiness to cry in the previous week.
Table 3

Categories of responses to question concerning precipitants of tearfulness from the Research Interview

\begin{tabular}{lc}
\hline Category & Number of responses \\
\hline Separation from and thoughts of fam- & 6 \\
ily, including bereavements & \\
Films or television & 6 \\
Talking about the situation & 4 \\
Acts of kindness by staff or friends & 3 \\
Trying to do something that used to be & 2 \\
easy, and failing & \\
Religion & 2 \\
\hline
\end{tabular}

Table 4

Categories of responses to question concerning strategies for coping with tearfulness from the Research Interview

\begin{tabular}{lc}
\hline Category & Number of responses \\
\hline Avoidance or escape (usually cogni- & 2 \\
tive), including active use of distraction & \\
Specific self control procedure & 2 \\
Self talk/positive thinking & 1 \\
Try hard/make an effort & 1 \\
Talking to family or friends & 1 \\
Talking to professionals & 1 \\
\hline
\end{tabular}

\subsection{Precipitants}

The 43 subjects who reported emotionalism-tearfulness generated 23 responses to the question concerning precipitants of this experience (question $b$ )). These were assigned to 6 emergent categories, with $88 \%$ agreement between raters for category assignment, summarised in Table 3.

\subsection{Coping strategies}

These 43 subjects were also able to generate 8 responses to the question concerning coping strategies which they found helpful (question c)). These were assigned to 6 emergent categories, with $93 \%$ agreement between raters for category assignment, summarised in Table .

\subsection{Relationship with other emotions}

Statistically significant correlations were obtained between Emotionalism-tearfulness ratings (question d)) and ratings for other interview items: with Sadness (tau $=0.38, p<0.001)$; Frustration $(\operatorname{tau}=0.28$, $p<0.01)$; Fear (tau $=0.25, p<0.01)$; and Worry (tau $=0.25, p<0.01)$. Emotionalism-tearfulness was not correlated with Apathy, Irritability, Inappropriate behaviour, Emotionalism-laughter, or Confusion.

The relation between reported Sadness and Emotionalism was further investigated by examining the simple 
endorsement of these experiences of (answering 'yes' to question a) and 'yes' to the question, 'I would like you to tell me if you have been low or sad' which occurred at a different point in the interview). These data are presented in Table 5.

\section{Table 5}

Contingency table illustrating relationship between Emotionalism and Sadness (from Research Interview), Chi squared $=7.641$ d.f. $p<0.01$

\begin{tabular}{lcc}
\hline & $\begin{array}{c}\text { Emotionalism } \\
\text { reported }\end{array}$ & $\begin{array}{c}\text { Emotionalism } \\
\text { not reported }\end{array}$ \\
\hline Sadness reported & 37 & 23 \\
Sadness not reported & 6 & 16 \\
\hline
\end{tabular}

It can be seen that most subjects who reported Emotionalism-tearfulness also reported Sadness. Only 6 subjects reported crying and also reported no feelings of Sadness. Four of these subjects described bursting into tears when angry and frustrated. 2 out of the total 43 described Emotionalism-tearfulness apparently unconnected with the experience of other emotions.

\subsection{Variables that predicted crying during the Research Interview or pre-interview procedure}

The target category contained 36 subjects. The total model was significantly predictive $\left(\chi^{2}=20.96,2\right.$ d.f., $p<0.001$ ) with only two contributing independent variables:

$$
\begin{array}{cc}
\text { Gender (female) } & \text { (Wald statistic }=13.72, \\
& 1 \text { d.f., } p<0.001) \\
\text { Focal right brain damage } & \text { (Wald statistic }=7.48, \\
& 1 \text { d.f., } p<0.01)
\end{array}
$$

\section{Discussion}

The prevalence rates of emotionalism reported in this study, whether on the basis of self report or observed behaviour, are high in comparison to previous estimates. This may be because of the definitions of emotionalism which were employed (subjects' own perception that they were crying or laughing 'too' easily or without sufficient cause; or overt crying behaviour when discussing their emotions with the investigator). No conditions concerning the uncontrollability, suddenness, meaningfulness or intensity of the behaviour were imposed. This was a considered decision because these qualities are difficult to define reliably, and varying degrees of emphasis have been placed on them in the literature. A rigid definition of the features of emotional- ism is premature in the absence of descriptive data from large groups of subjects with a range of neurological disorders.

The precipitants of emotionalism, as described by subjects in this study, were separation from and thoughts of family, films or television, talking about the situation, acts of kindness, and religion. Some subjects also vividly described crying in situations where they were frustrated. These findings are entirely consistent with Allman's study of stroke patients [3], and demonstrate that his conclusions can be extended to a sample of brain injured subjects of mixed aetiology. The words used to describe emotionalism by subjects were diverse. Several subjects described a kind of sentimentality, or exaggerated affective response across a range of positive and negative emotions:

'I've always been a bit that way, but more now. I cry when I'm frightened, when my husband left and I felt sad, and when I was chuffed to bits at going to the loo alone.'

'All these emotions can cause me to cry.'

A number of subjects reported that they could bring crying under control with relative ease, by concentrating hard and trying to stop, or by the use of active distraction. One subject described the crying as uncontrollable but time limited. It is likely that the subjects who declined to be interviewed because of tearfulness and distress were experiencing prolonged bouts of crying that were more difficult to control.

Only about half of the subjects who reported Emotionalism-tearfulness (26\% of the total sample) described it as causing them significant personal distress. Thus, many subjects experienced emotionalism as a minor nuisance rather than as a major problem. This may again be related to the inclusive definition of emotionalism employed in this study.

There was a clear association between Emotionalismtearfulness and Sadness, evident both in terms of a categorical measure (co-occurrence) and a dimensional measure (correlation). This is consistent with the findings of House and colleagues that emotionalism in their sample was not meaningless, and was usually accompanied by negative affect [9]. In the present sample 'pure' Emotionalism was rare, and the more general crying observed may be better described as disinhibited behaviour signifying affective experience. Thus, the emotionalism displayed by these subjects was meaningful, associated with negative affect, but only resulted in major distress for about half the subjects who reported it. Of the 43 subjects who reported Emotionalismtearfulness, only nine $(20 \%)$ were diagnosed as de- 
pressed by the unit psychiatrist (6 as major depressive disorder, three as adjustment disorder with depressed mood). Therefore the majority of subjects who reported or displayed emotionalism (and sadness) were not thought to be depressed by the clinical team that cared for them.

The logistic regression analysis indicated that only female gender and focal right sided brain damage were predictive of crying when interviewed. Diagnosis itself was not predictive, nor were degree of impairment or disability. The association of crying with being female may have gone unnoticed in previous studies because crying is less likely to be judged as abnormal in females in our culture, and because fewer females than males are affected by acquired brain injury. It is possible that females may have to show extremes of this behaviour before it is judged to be clinically significant. The finding that focal right sided brain damage was predictive of crying in the interview situation provides some support for right hemisphere theories of emotionalism [19], but contradicts the findings of House and colleagues [9]. However, it should be interpreted with some caution, as detailed neuroimaging data were not available, and it is possible that the right sided lesions were larger than the left sided lesions in this sample.

\section{Conclusions}

This study has demonstrated that self reported increased readiness to cry, and crying behaviour in a standard private interview setting, were common in a mixed sample of subjects with severe acquired brain injury of mixed aetiology. Focal lesion to the right hemisphere and female gender were identified as possible vulnerability factors.

The behaviour occurred in response to clearly identifiable and meaningful precipitants, consistent with those reported in studies of stroke patients, demonstrating that their findings can be applied to other neurological samples. It was clearly associated with negative affect but only symptomatic of depression in a minority of cases. The intensity, controllability, and duration of the emotional behaviour, and the degree of associated distress were highly variable. This suggests that it is more appropriate to describe emotionalism as a neurobehavioural dimension rather than as a syndrome. Where a patient shows behavioural extremes of intensity, uncontrollability, with a lack of connection with environmental circumstances it may be appropriate to invoke the syndrome concept. However, these cases are rare. This adds a further challenge to the idea of emotionalism as a discrete stereotyped syndrome with a specific biological explanation, and suggests the need for further research into its psychological associations and response to treatment.

In general clinical practice it is important to be able to detect emotionalism (and this study suggests that it may be given less attention in females). It also is important to ascertain the precise degree of distress involved. This may not be as profound as it appears, and information to this effect can be very reassuring for family members and carers. These people can be encouraged to respond in a way that is acceptable to the patient, supporting his or her own coping strategies and engendering a sense of control (for instance changing the topic of conversation or pressing on regardless, leaving the room, having tissues to hand, acknowledging the distress felt etc.). Following this study a leaflet on emotionalism for use with patients, families, and carers was introduced at the unit, and has proved helpful in a number of cases. Use of the leaflet saves the patient having to explain him or herself to others, and can make certain social situations less threatening, thus reducing the social isolation that can be a consequence of emotionalism.

The degree of distress associated with emotionalism, together with its persistence, is also likely to influence decisions on the use of drug treatments. However, even when medication is used it should also be acknowledged that the emotionalism may reflect meaningful concerns of the patient, rather than merely the end point of a biological process. These concerns may themselves be amenable to practical or psychological approaches, and thus be targeted within treatment programmes.

This study was carried out in accordance with the Central Oxford Research 1 Ethics Committee - Approved Study 93.085 .

\section{Acknowledgement}

Thanks to Professor Allan House, University of Leeds, for helpful comments on this manuscript.

\section{References}

[1] P. Allman, R.A. Hope and C.G. Fairburn, Emotionalism following brain damage: a complex phenomenon, Postgraduate Medical Journal 66 (1990), 818-812. 
[2] P. Allman, Depressive disorders and emotionalism following stroke, International Journal of Geriatric Psychiatry 6 (1991), 377-383.

[3] P. Allman, Emotionalism following brain damage, $B e$ havioural Neurology 4 (1991), 57-62.

[4] P. Allman, Drug treatment of emotionalism following brain damage, Journal of the Royal Society of Medicine 85 (1992), 423-424.

[5] C. Collin, D.T. Wade, S. Davis and V. Horne, The Barthel A.D.L. Index: a reliability study, International Disability Studies 10 (1988), 61-63.

[6] P.M. Enderby, V.A. Wood, D.T. Wade and R. Langton Hewer, The Fenchay Aphasia Screening Test: a short, simple test for aphasia appropriate for non-specialists, International Rehabilitation Medicine 8 (1986), 166-170.

[7] J. Gallagher, Pathological laughter and crying in amyotrophic lateral sclerosis, Acta Neurologia Scandinavica 80 (1989), 114-117.

[8] H.C. Hanger, Emotionalism after stroke, Letter to Lancet 342 (1993), 1235-1236.

[9] A. House, M. Dennis, A. Molyneux, C. Warlow and K. Hawton, Emotionalism after stroke, British Medical Journal 298 (1989), 991-994.

[10] A. House, M. Dennis, L. Mogridge, C. Warlow, K. Hawton and L. Jones, Mood disorders in the first year after stroke, British Journal of Psychiatry 158 (1991), 83-92.

[11] F. Lebert, F. Pasquier, M. Steinling and H. Petit, Affective disorders related to S.P.E.C.T, patterns in alzheimer's disease: a study of emotionalism, International Journal of Geriatric Psychiatry 9 (1994), 327-329.

[12] A. Lieberman and D.F. Benson, Control of emotional expression in pseudobulbar palsy, Archives of Neurology 34 (1977), 717-719.

[13] J. McGrath and L. Adams, Patient-centered goal planning: a systemic psychological therapy? Topics in Stroke Rehabilitation 6 (1999), 43-50.

[14] D.M. McNair, M. Lorr and L.F. Droppleman, EDITS manual for the Profile of Mood States, Educational and Industrial Service, San Diego, 1981.

[15] D.L. Nyenhuis, R.A. Stern, C. Yamamoto, T. Luchetta and J.E. Arruda, Standardization and validation of the Visual Analog Mood Scales, The Clinical Neuropsychologist 11 (1997), 407415.

[16] V.M. Parker, D.T. Wade and R. Langton Hewer, Loss of arm function after stroke: measurement, frequency and recovery, International Rehabilitation Medicine 8 (1986), 69-73.

[17] K. Poeck, Pathophysiology of emotional disorders associated with brain damage, in: Handbook of Clinical Neurology, (Vol. 3), P.J. Vinken and G.W. Bruyn, eds, North Holland Publishing, Amsterdam, 1969.

[18] E.D. Ross, Modulation of affect and nonverbal communication by the right hemisphere, in: Principles of Behavioural Neurology, M. Mesulam, ed., F.A. Davis, Philadelphia, 1985.

[19] E.D. Ross and R.S. Stewart, Pathological display of affect in patients with depression and right frontal brain damage, Journal of Nervous and Mental Disease 175 (1987), 165-172.
[20] P.K. Sethi and T.S. Rao, Gelastic, quiritanous, and cursive epilepsy, Journal of Neurology, Neurosurgery and Psychiatry 39 (1976), 323-828.

[21] R.L. Sloan, W. Brown and B. Pentland, Fluoxetine as a treatment for emotional lability after brain injury, Brain Injury 6 (1992), 315-319.

[22] D. Surridge, An investigation into some psychiatric aspects of multiple sclerosis, British Journal of Psyhciatry 115 (1969), 749-764.

[23] G. Teasdale, G. Murray, L. Parker and B. Jennet, Adding up the Glasgow Coma Scale, Acta Neurochirurgica 28 (1979), $12-16$.

[24] S.A.K. Wilson, Some problems in neurology - pathological laughing and crying, Journal of Neurology and Neuropathology 16 (1924), 299-333.

\section{Appendix: Independent variables used in stepwise logistic regression procedure}

1. Time since onset

2. Age

3. Gender

4. Diagnosis (stroke, head injury, subarachnoid haemorrhage, other)

5. Lesion location (right, left, anterior, posterior cerebral lesion noted on scan)

6. Deepest coma since onset (Glasgow Coma Scale [23])

7. General motor impairment (Motricity Index [16])

8. Ataxia

9. Contractures

10. Dysphagia

11. Epilepsy

12. Sensory impairment

13. Major pain

14. Cognitive impairment (amnesia, apraxia, visuoperceptual impairment)

15. Premorbid psychiatric history

16. Premorbid alcohol abuse

17. Fall since onset

18. Major life event in last 6 months

19. Functional ability level (Barthel Index [5])

20. Functional communication (Frenchay Aphasia Screening Test [6]) 


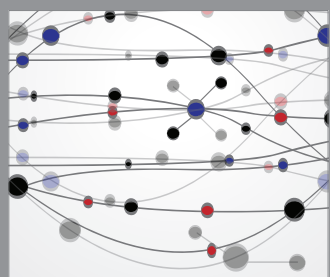

The Scientific World Journal
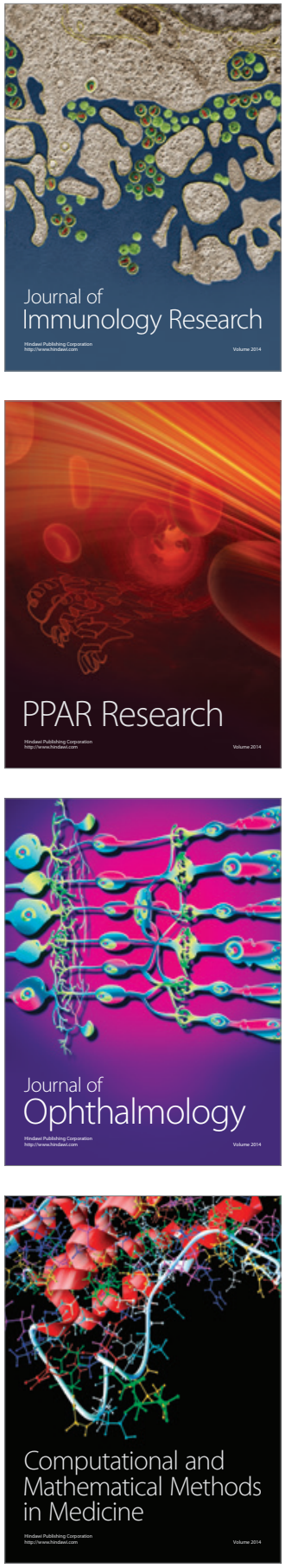

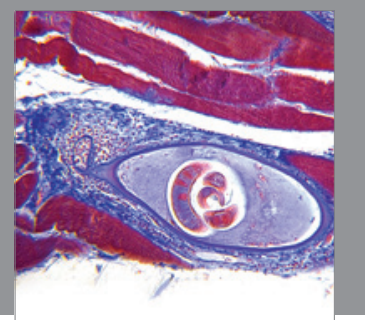

Gastroenterology

Research and Practice
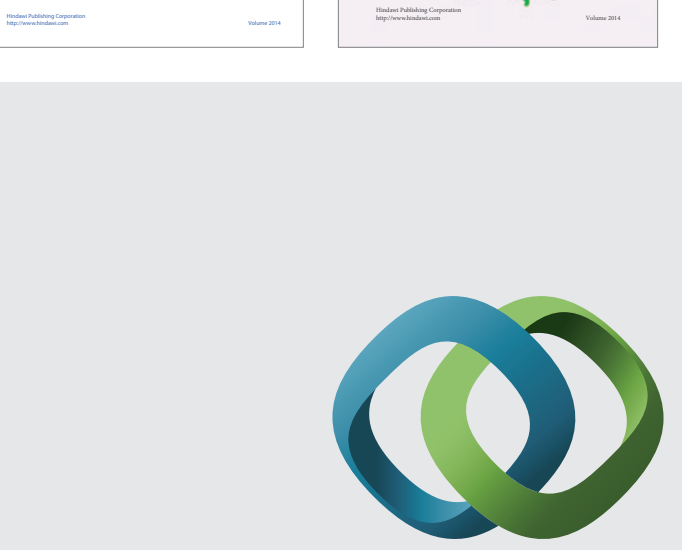

\section{Hindawi}

Submit your manuscripts at

http://www.hindawi.com
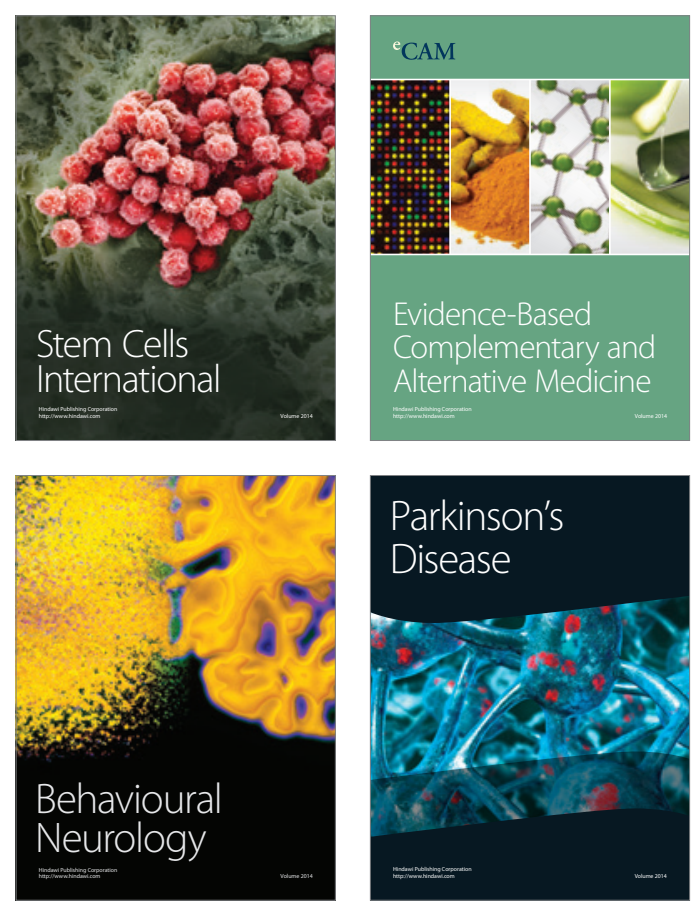

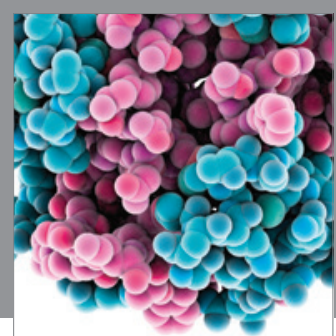

Journal of
Diabetes Research

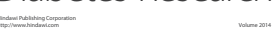

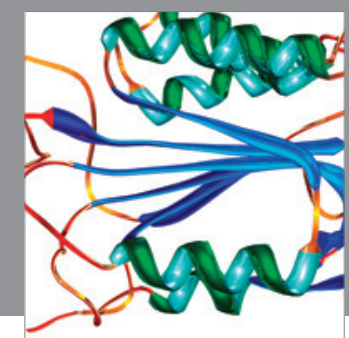

Disease Markers
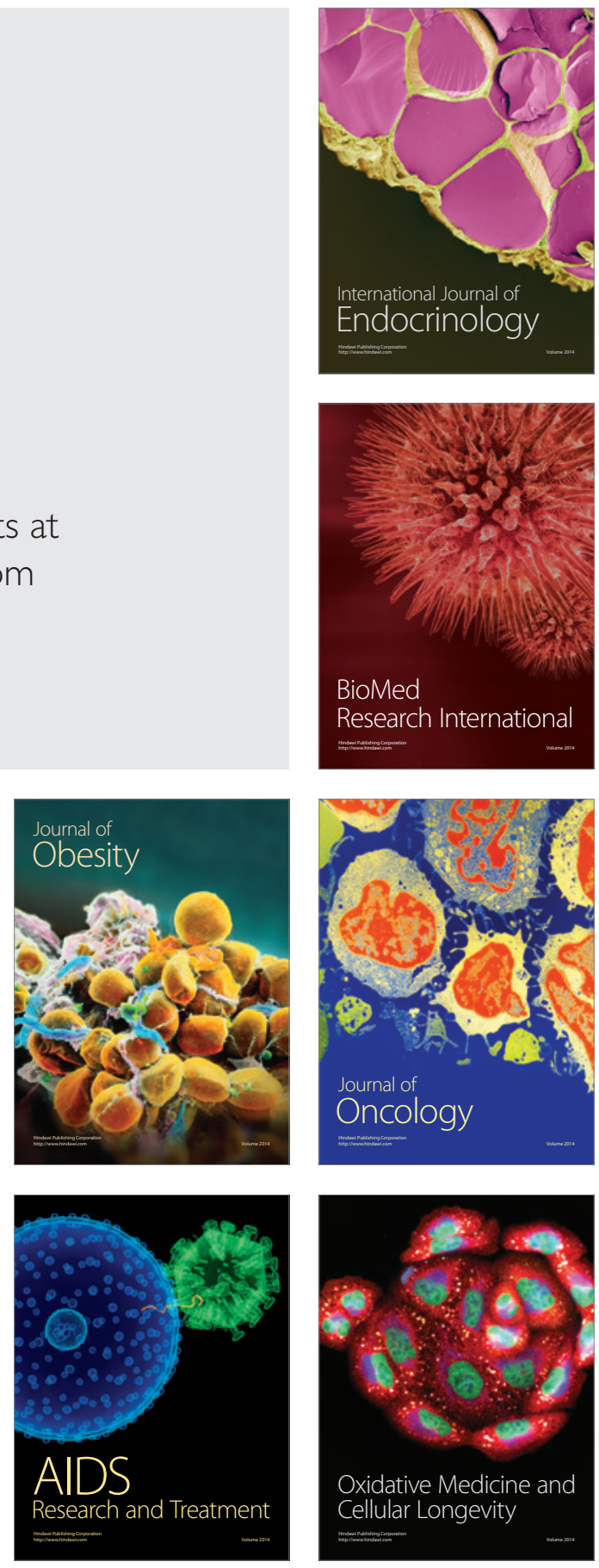